\title{
Progress in drug treatment of older patients with chronic heart failure
}

\author{
Siting Zhang ${ }^{\mathrm{a}}$, Feng Liu $^{\mathrm{a},{ }^{*}}$ \\ ${ }^{a}$ National Key Clinical Department of Gerontology, Guangzhou First People's Hospital, School of Medicine, South China \\ University of Technology, Guangzhou 510180, China.
}

\begin{abstract}
Chronic heart failure(CHF) is a serious public health problem in the world, which occurs mostly in the elderly, with extremely high morbidity and mortality. The physiological changes in the cardiovascular system caused by aging are an independent risk factor for CHF. For this reason, effective drug treatment is often used to improve the clinical symptoms and quality of life of older CHF patients, and ultimately achieve the goal of reducing hospitalization rate and cardiovascular mortality. This article describes recent progress of drug therapy for older patients in chronic heart failure.

Keywords: Aging, older patients, chronic heart failure, drug therapy
\end{abstract}

Chronic heart failure refers to the clinical symptoms of various cardiovascular diseases that progress to the end stage. Among them, coronary heart disease and hypertension are the main causes, and it is also the main cause of death in older patients with cardiovascular disease [1]. As the population ages, the global prevalence and mortality of chronic heart failure are still on the rise. It is estimated that in developed countries, more than $10 \%$ of older $\mathrm{CHF}$ patients are 70 or more years of age [2]. How to effectively improve the heart function of older patients with chronic heart failure, improve the quality of life, reduce the incidence of acute attacks, control the progression of the disease, and extend the survival time has become a hot topic in the clinical world. Now it is expounded from two aspects: the pathogenesis of chronic heart failure and the progress of drug treatment.

\section{Pathogenesis of chronic heart failure}

CHF is a group of clinical syndromes that causes changes in ventricular structure or function due to various reasons, and ultimately results in systolic or diastolic dysfunction.

\footnotetext{
* Corresponding author: Feng Liu

Mailing address: National Key Clinical Department of Gerontology, Guangzhou First People's Hospital, School of Medicine, South China University of Technology, Guangzhou 510180, China.

Email: eyliufeng@scut.edu.cn
}

Received: 28 November 2020 / Accepted: 20 December 2020
Its clinical manifestations are mainly dyspnea, fatigue and fluid retention (pulmonary congestion, systemic congestion and peripheral edema) $[3,4]$. Neuroendocrine hyperactivation and ventricular remodeling involving a variety of cytokines are currently the main pathogenesis of CHF.

\section{Neuroendocrine system}

The renin-angiotensin-aldosterone system(RAAS) has been shown to play a key role in the occurrence and development of CHF [5]. RAAS is a fluid regulation system in the body, which plays an important role in the maintenance of normal cardiovascular development, stable function, electrolyte and fluid balance $[6,7]$. When renin is released in the blood and RAAS is initiated, angiotensinogen is hydrolyzed into angiotensin I under the action of renin to form angiotensin II, causing vasoconstriction, water and sodium retention, and ventricular hypertrophy, myocardial hyperplasia, reducing cardiac output [5].

\section{Ventricular remodeling}

Under pathophysiological stimulation, a variety of molecules and cells are involved in ventricular remodeling, including fibroblasts (promoting fibrosis), vascular smooth muscle cells (promoting vascular stiffness), vascular endothelial cells (promoting endothelial dysfunction) and leukocytes (causing inflammation) [8]. Ventricular remodeling is a repair process that plays a certain compensatory role in CHF in the early stage. If it is not controlled, it will lead to cardiomyocyte hypertrophy, ventricular wall thinning, ventricular cavity expansion, and excessive extracellular matrix accumulation leading to heart muscle fibrosis eventually to $\mathrm{CHF}[9]$. 
Progress in drug treatment of chronic heart failure

Improving clinical symptoms and quality of life by preventing or reversing cardiac remodeling is a major goal of the treatment of chronic heart failure, and an important way to reduce rehospitalization and mortality. The core of CHF drug treatment mainly includes beta-receptor blockers, RAAS inhibitors (ie, angiotensin-converting enzyme inhibitor ACEI and angiotensin II receptor antagonist $\mathrm{ARB}$ ) and aldosterone receptor antagonists, which are still recommended as first-line drugs (Class I recommendations) by domestic and foreign guidelines [1, 3, 10]. The combination of these three drugs is called the "Golden Triangle" in the 2014 China Heart Failure Diagnosis and Treatment Guidelines [11]. It can effectively improve symptoms and quality of life, reduce the risk of death, hospitalization, and sudden death. It is tolerated very well by older CHF patients [12]. In addition to the aforementioned prognostic-improving drugs, diuretics and digitalis drugs are also very important in improving symptoms. With the continuous advancement of medical technology, the effectiveness and safety of a variety of new drugs in the treatment of CHF have been initially confirmed, providing new ideas for the treatment of CHF patients.

\section{Angiotensin receptor-enkephalinase inhibitor(ARNI)}

Sacubitrilvalsartan (LZC696) is the world's first ARNI. It is a new type of drug for the treatment of heart failure composed of valsartan(ARB) and sacubitril(NEPI) in a ratio of $1: 1$, which can inhibit the RAAS system and regulate the natriuretic peptide system [13]. The PARADIGMHF study [14] is a study including China, which aims to compare the basic treatment of heart failure with the addition of sacubitrilvalsartan $200 \mathrm{mg}$ twice a day and enalapril $10 \mathrm{mg}$ twice a day, a multi-center, prospective, randomized, double-blind study on the mortality and rehospitalization rate of 8,399 CHF patients with NYHA IIIV and left ventricular ejection fraction (LVEF) $\leq 35 \%$ [15] After an average follow-up of 27 months, the results of the study showed that compared with enalapril, the risk of cardiovascular death in the sacubitrilvalsartan group was reduced by $20 \%(\mathrm{P}<0.001)$, and the risk of hospitalization due to heart failure decrease by $21 \%(\mathrm{P}<0.001)$. The risk of all-cause death in the sacubitrilvalsartan group was $16 \%$ lower than that in the enalapril group $[16,17]$. Therefore, the 2017 ACC/AHA/HFSA Heart Failure Management Guidelines recommends that HFrEF patients with $\mathrm{LVEF} \leq 35 \%$ can be treated with ARNI, and it is recommended for all NYHA heart function class II-III patients, who can tolerate ACEI/ARB treatment and have chronic symptomatic HFrEF be treated with ARNI to reduce morbidity and mortality, and receive a Class I recommendation [1].

\section{Specific inhibitor of If current in sinoatrial node}

Ivabradine can specifically inhibit cardiac sinus node pacing current, slow down heart rate, and reduce myocardial oxygen consumption. At the same time, it does not have the blood pressure lowering effect of beta blockers, thereby improving ventricular remodeling and significantly improving CHF patients quality of Life. The SHIFT study is a randomized, double-blind, placebo-controlled study that included 6558 NYHA II-IV patients with left ventricular ejection fraction $\leq 35 \%$ and sinus heart rate $\geq 70$ beats/ min. They were randomly divided into Ivabradine group or the comfort crew. After 22.9 months of follow-up, the results showed that the relative risk of cardiovascular death and worsening heart failure in the ivabradine group was reduced, and the left ventricular function and quality of life of the patients were significantly improved, reversing ventricular remodeling, and the number of serious adverse events was lower than that in the placebo group $[18,19]$. The 2016 European Society of Cardiology (ESC) heart failure guidelines pointed out that patients with LVEF $\leq 35 \%$ and sinus heart rate $\geq 70$ beats/min should consider using ivabradine after the target dose or maximum tolerated dose of beta blockers, ACEI or ARB and aldosterone receptor antagonists [10].

\section{Vasopressin V2 receptor antagonist}

Tolvaptan inhibits water reabsorption by binding to the V2 receptor, but does not increase sodium excretion, so it is often used in the treatment of CHF with hyponatremia. The EVEREST study consisted of two identical prospective, randomized, double-blind, placebo-controlled trials. 4,133 hospitalized patients with heart failure were randomly divided into tolvaptan group or placebo group. The results showed the clinical effect of tolvaptan group was significantly improved, and the weight loss was more significant. The incidence of serious adverse reactions in the two groups was similar, with no significant renal failure or hypotension, but it failed to reduce the mortality and hospitalization rate of patients with heart failure [20]. In a retrospective study in Japan, it was also shown that the addition of small doses of tolvaptan to CHF patients with hyponatremia in a short period of time was well tolerated with a low incidence of main side effects, which is useful for correcting severe hyponatremia [21].

\section{Sodium-glucose cotransporter 2 inhibitor}

SGLT-2 inhibitor is a new type of oral hypoglycemic agent. Its basic function is to block the SGLT-2 transporter in the proximal tubules of the kidney, inhibit the reabsorption of glucose by the renal tubules, and increase the urine osmotic pressure, thereby promoting the excretion of urine sugar and sodium excretion. The EMPA-REG OUTCOME study showed that compared with the placebo group, type 2 diabetes patients with cardiovascular disease who used the SGLT-2 inhibitor Empagliflozin could reduce the incidence of the main composite cardiovascular endpoint by $14 \%$, all-cause mortality by $38 \%$, and hospitalization risk of heart failure by $35 \%$ [22]. The results of the DAPA-HF study showed that on the basis of standard treatment for heart failure, the addition of SGLT-2 inhibitor Dapagliflozin can significantly reduce the risk of cardiovascular death or worsening of heart failure, reduce the 
risk of cardiovascular death, and can significantly reduce the risk of all-cause death [23].The 2020 Canadian Cardiology Society (CCS) and Canadian Heart Failure Society (CHFS) heart failure guidelines recommended that SGLT2 inhibitors should be applied to patients with mild to moderate heart failure with $\mathrm{LVEF} \leq 40 \%$ and complicated with type 2 diabetes to relieve heart failure symptoms, improve quality of life and reduce the risk of hospitalization and cardiovascular death [24].

\section{Soluble guanylate cyclase sGC stimulator}

Cyclic guanosine phosphate(cGMP) is a key signaling molecule involved in the regulation of vascular tension, proliferation, fibrosis and inflammation. Riociguat directly stimulates sGC in a non-nitric oxide way, increasing the sensitivity of sGC to nitric oxide, leading to an increase in cGMP production, thereby promoting vasodilation [25]. Riociguat is the world's first drug approved for the treatment of pulmonary hypertension, and its role in the field of heart failure still needs further research to verify. The VICTORIA study included 5050 CHF patients with NYHA II-IV and LVEF $\leq 45 \%$, which was a randomized, double-blind, placebo-controlled phase III clinical trial. It was confirmed that the addition of Vericiguatl on the basis of standard treatment could reduce the composite endpoint of cardiovascular death or hospitalization for heart failure in CHF patients by $10 \%$ [26].

\section{Conclusion}

Chronic heart failure has a high morbidity and mortality rate, which poses a great threat to the safety and quality of life of older patients. However, drug treatment is still the main clinical approach. The clinical efficacy, and compatibility of various new therapeutic drugs still need more research to confirm and achieve scientific medication according to the guidelines, but also to formulate targeted treatment plans based on the individualization of the older patients. It is believed that with an increase in clinical research, more effective drugs will become available, and great progress will be made in drug treatment of older patients with chronic heart failure.

\section{References}

1. Clyde W, Yancy CW, Jessup M, et al. 2017 ACC/AHA/HFSA Focused Update of the 2013 ACCF/AHA Guideline for the Management of Heart Failure: A Report of the American College of Cardiology/American Heart Association Task Force on Clinical Practice Guidelines and the Heart Failure Society of America. J Am Coll Cardiol, 2017, 70(6) 776-803.

2. Ambrosy AP, Gheorghiade M, Chioncel O, Mentz RJ, Butler J. Global Perspectives in Hospitalized Heart Failure: Regional and Ethnic Variation in Patient Characteristics, Management, and Outcomes. Current Heart Failure Re- ports, 2014, 11(4): 416-427.

3. Heart Failure Group of Cardiovascular Branch of Chinese Medical Association, Heart Failure Professional Committee of Chinese Medical Doctor Association, Editorial Committee of chinese journal of cardiology. Chinese Heart Failure Diagnosis and Treatment Guide 2018. Chinese Journal of Cardiovascular Disease, 2018, 46(10):760-789.

4. William TA, Mitchell AP, Mona F, et al. Standardized Definitions for Evaluation of Heart Failure Therapies: Scientific Expert Panel From the Heart Failure Collaboratory and Academic Research Consortium. JACC: Heart Failure, 2020,8(12):961-972.

5. Mollace V, Gliozzi M, Capuano A, et al. Modulation of RAAS-natriuretic peptides in the treatment of HF: old guys and newcomers.International Journal Cardiology, 2017, 226:126-131.

6. Bomback AS, Toto R. Dual blockade of the renin-angiotensin-aldosterone system: beyond the ACE inhibitor and angiotensin-II receptor blocker combination. American Journal Hypertension, 2009, 22(10):1032-1040.

7. James J. The renin-angiotensin aldosterone system: pathophysiological role and pharmacologic inhibition. Journal of managed care pharmacy, 2007, 13(8):9-20.

8. Min X, Jana SB, Joseph AH. Pathological Ventricular Remodeling: Mechanisms: Part 1 of 2. Circulation, 2008, 128(4):388-400.

9. Hill JA, Olson EN. Cardiac plasticity. N Engl J Med, 2008, 358(13):1370-1380.

10. Ponikowski P, Voors AA, Anker SD, et al. 2016 ESC Guidelines for the diagnosis and treatment of acute and chronic heart failure. Eur Heart J, 2016, 37(27):2129-2200.

11. Chinese Medical Association Cardiovascular Diseases Branch, Chinese Journal of Cardiovascular Diseases Editorial Committee. Chinese Heart Failure Diagnosis and Treatment Guidelines 2014. 2014, 42(2):3-10.

12. Burnett H, Earley A, Voors AA, et al. Thirty Years of Evidence on the Efficacy of Drug Treatments for Chronic Heart Failure With Reduced Ejection Fraction: A Network Meta-Analysis. Circulation: Heart Failure, 2017, 10(1):e003529.

13. Docherty KF, Mcmurray JJV. Angiotensin receptorneprilysin inhibitors: A new paradigm in heart failure with reduced ejection fraction. International Journal of Cardiology, 2019, 281: 179-185.

14. Jessup M, Fox KA, Komajda M,et al.PARADIGM-HF: the experts' discussion. The New England Journal of Medicine, 2014, 371(11) : e15.

15. Balmforth C, Simpson J, Shen L, Jhund PS, Lefkowitz M, Rizkala AR, Rouleau JL, Shi V, Solomon SD, Swedberg K, Zile MR, Packer M, McMurray JJV. Outcomes and Effect of Treatment According to Etiology in HFrEF:An Analysis of PARADIGM-HF. JACC: Heart Failure, 2019, 7(6): 457-465.

16. MCMURRAY JJ, PACKER M, DESAI A S, et al.Dual angiotensin receptor and neprilysin inhibition as an alternative to angiotensin-converting enzyme inhibition in patients with chronic systolic heart failure: rationale for and design of the prospective comparison of ARNI 
with ACEI to determine impact on global mortality and morbidity in heart failure trial(PARADIGM-HF). Europan Journal Heart Failure, 2013, 15(9): 1062-1073.

17. McMurray JJ, Packer M, Desai AS, et al.Angiotensin-neprilysin inhibition versus enalapril in heart failure. The New England Journal Medicine, 2014, 371(11) :993-1004.

18. Swedberg K, Komajda M, Böhm M, et al. Ivabradine and outcomes in chronic heart failure (SHIFT): a randomised placebo-controlled study. Lancet, 2010, 376(9744):87585.

19. Tardif JC, O'Meara E, Komajda M, et al. Effects of selective heart rate reduction with ivabradine on left ventricular remodelling and function: results from the SHIFT echocardiography substudy. Eur Heart J, 2011, 32(20):250715.

20. Konstam MA, Gheorghiade M, Burnett JC, et al. Effects of oral tolvaptan in patients hospitalized for worsening heart failure: the EVEREST Outcome Trial. JAMA, 2007,297(12):1319-31.

21. Sağ S, Aydın Kaderli A, Yıldız A, et al. Use of tolvaptan in patients hospitalized for worsening chronic heart failure with severe hyponatremia: The initial experience at a single-center in Turkey. Turk Kardiyol Dern Ars, 2017, 45(5):415-425.

22. Fitchett D, Zinman B, Wanner C, et al. Heart failure outcomes with empagliflozin in patients with type 2 diabetes at high cardiovascular risk: results of the EMPA-REG OUTCOME® trial. Eur Heart J, 2016, 37(19):1526-34.

23. Mcmurray JJV, Demets DL, Inzucchi SE, et al. The Dapagliflozin And Prevention of Adverse-outcomes in Heart Failure (DAPA-HF) trial: baseline characteristics. European Journal of Heart Failure, 2019, 21(11).

24. Eileen OM, Michael M, Michael C, et al. CCS/CHFS Heart Failure Guidelines: Clinical Trial Update on Functional Mitral Regurgitation, SGLT2 Inhibitors, ARNI in HFpEF, and Tafamidis in Amyloidosis. Can J Cardiol, 2020, 36(2): 159-169.

25. Conole D, Scott LJ. Riociguat: first global approval. Drugs, 2013, 73(17):1967-75.

26. Armstrong PW, Roessig L, Patel MJ, et al. A Multicenter, Randomized, Double-Blind, Placebo-Controlled Trial of the Efficacy and Safety of the Oral Soluble Guanylate Cyclase Stimulator: The VICTORIA Trial. JACC Heart Fail, 2018, 6(2):96-104. 